\title{
Passive Monitoring at Home: A Pilot Study in Parkinson Disease
}

\author{
Zachary Kabelac $^{a} \quad$ Christopher G. Tarollib, c Christopher Snyder ${ }^{c}$ \\ Blake Feldman ${ }^{c} \quad$ Alistair Glidden ${ }^{c}$ Chen-Yu Hsu ${ }^{a}$ Rumen Hristov ${ }^{a}$ \\ E. Ray Dorsey ${ }^{c}$ Dina Katabi ${ }^{a}$ \\ aDepartment of Computer Science and Artificial Intelligence, Massachusetts Institute of \\ Technology, Cambridge, MA, USA; ${ }^{b}$ Department of Neurology, University of Rochester \\ Medical Center, Rochester, NY, USA; ${ }^{c}$ Center for Health \& Technology, University of \\ Rochester Medical Center, Rochester, NY, USA
}

\author{
Keywords \\ Parkinson disease · Sleep disorders · Gait speed · Quality of life
}

\section{Abstract}

We conducted a pilot study using a passive radio-wave-based home monitor in individuals with Parkinson disease (PD) with a focus on gait, home activity, and time in bed. We enrolled 7 ambulatory individuals to have the device installed in the bedroom of their homes over 8 weeks and performed standard PD assessments at baseline. We evaluated the ability of the device to objectively measure gait and time in bed and to generate novel visualizations of home activity. We captured 353 days of monitoring. Mean gait speed $(0.39-0.78 \mathrm{~m} / \mathrm{s})$, time in bed per day (4.4-12.1 h), and number (1.4-5.9) and duration (15.0-49.8 min) of nightly awakenings varied substantially across and within individuals. Derived gait speed correlated well with the Movement Disorder Society-Unified Parkinson's Disease Rating Scale total $(r=-0.88$, $p=0.009)$ and motor sub-score $(r=-0.95, p=0.001)$. Six of the seven participants agreed that their activity was typical and indicated a willingness to continue monitoring. This technology provided promising new insights into the home activities of those with PD and may be broadly applicable to other chronic conditions.

(c) 2019 The Author(s)

Published by S. Karger AG, Basel

Zachary Kabelac and Christopher G. Tarolli are co-first authors.

Zachary Kabelac, PhD

MIT Computer Science and Artificial Intelligence Lab

32 Vassar Street, 32-268

Cambridge, MA 02139 (USA)

E-Mail zek@mit.edu 
Kabelac et al.: Passive Monitoring at Home: A Pilot Study in Parkinson Disease

\section{Introduction}

Traditional monitoring of Parkinson disease (PD) has relied on self-reporting of symptoms or subjective episodic assessments performed in the clinic. Passive home monitoring can enhance clinical assessments by objectively capturing real-world function in patients with chronic disease [1-3]. The Emerald device is a novel radio-wave-based home activity monitor that passively collects data on participant motion in a monitored space without requiring direct interaction with the system. The device requires no collection of identifiable participant data [4], is small and easy to install [5], and can differentiate individuals in a monitored space [2]. Previous studies have demonstrated the ability of the device to monitor position and gait speed in the clinic and at home [6-8]. In this pilot study, we installed the Emerald device in the homes of individuals with PD to explore the relationship between traditional measures in the constrained standardized clinic and Emeraldderived measures collected uncontrolled at home. We sought to establish proof of concept of a novel method to characterize features of PD at home with a focus on time in bed, home activity, and gait.

\section{Methods}

\section{Emerald Device}

Researchers at the Massachusetts Institute of Technology developed a radio-wavesensing device called Emerald for passive patient monitoring. The system emits ultra-lowpower radio signals that reflect off individuals within a range, approximately 1,200 square feet, and return to the device. As individuals move and their reflected radio waves change, the device detects these changes to extract the position of the individual over time. Using the individual's position relative to the device, activity in the home and time in bed are computed based on the time spent by the individual in defined areas of interest, such as the bedroom, bathroom, bed, and hallway. Gait speed and other features of participant movement are derived from changes in position over time. Additional details of the analytics used to derive the participant's position and gait speed have been previously published [6-8].

Accuracy of the device has been validated against a VICON Motion Tracking System in individuals from age 20 to 83, completing thousands of experiments in the lab and at home. The device has been shown to accurately measure an individual's position to within $15 \mathrm{~cm}[6$, 8] and gait speed to within $0.025 \mathrm{~m} / \mathrm{s}$ [7]. Emerald can be installed in less than $1 \mathrm{~h}$ and hangs on the wall like a picture (Fig. 1). Encrypted data are transferred via WiFi to a secure fileserver hosted by Amazon Web Services (https://aws.amazon.com/). Monitored individuals are identified based on movement patterns that start or end at their position in bed. Those with a bed partner carry an accelerometer "tag" to identify themselves from others.

\section{Procedures}

We recruited, and obtained written consent from, ambulatory individuals with PD and a home WiFi connection around Rochester, NY, USA, to have the device installed in the bedroom of their home. During the installation, the boundaries of areas of interest in the home (e.g. bed, doorways) were measured with respect to the position of the device to create a schematic of the monitored space. We collected data for 8 weeks and asked participants to keep a Hauser diary [9] of motor status (medications working [ON] vs. not working [OFF]) for one day each week. Participants also completed typical PD assessments at baseline including the Movement Disorder Society-Unified Parkinson's Disease Rating Scale (MDS-UPDRS) parts I-IV [10], 
Fig. 1. Emerald radio-wave-sensing device installed at home (green box).

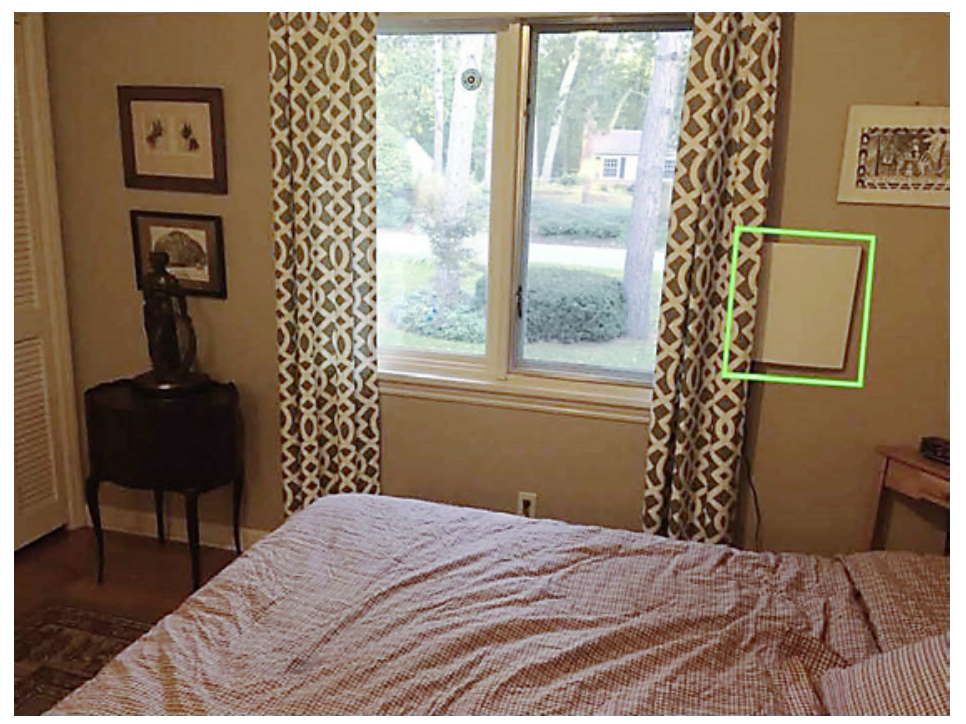

Hoehn and Yahr [11], Parkinson's Disease Sleep Scale (PDSS) [12], and Montreal Cognitive Assessment [13]. The Institutional Review Boards at the University of Rochester and Massachusetts Institute of Technology approved all study procedures.

\section{Outcomes}

We evaluated the ability of the device to measure participant time in bed, activity in the home, and gait speed based on absolute position and change in position relative to the Emerald device; we analyzed these data descriptively. We estimated a circadian schedule by calculating intraday variability in participant time in bed as a proxy for rest-activity schedules. The measure is calculated as the total difference (in h) of times in bed between consecutive days. Lower intraday variability values indicate greater consistency in time of rest and activity periods from day to day. For example, getting into bed for sleep at 10 p.m. and getting out of bed at 6 a.m. on consecutive days would equal a value of 0 [14]. We created visualizations of activity in the home and in bed by superimposing device-derived position in the home over the schematic of the monitored space.

We evaluated the correlation between device-derived gait speed and traditional measures of PD severity. We compared each participant's average gait speed across the entire monitoring period to the baseline MDS-UPDRS total and part III (motor sub-score) using a Pearson correlation. We evaluated the ability to detect response to dopaminergic therapy (a key feature of PD) by comparing the average device-derived gait speed across all OFF periods as reported on the Hauser diary to the average device-derived gait speed across all Hauserreported ON periods. We assessed significance using a one-tailed Wilcoxon signed-rank test $(\alpha=0.05)$.

Gait speed varies substantially based on the task being carried out [4], with a single gait speed measurement at home unlikely to adequately reflect overall functional ability. In order to account for this variability, we evaluated the optimal averaging window duration to capture an inclusive set of gait speed measurements to reflect functional ability, while minimizing monitoring time. We secondarily calculated the minimal detectable change with $95 \%$ confidence [15] in mean gait speed over the derived averaging window to evaluate the sensitivity of Emerald to detecting changes in gait speed (online suppl. Material; for all online suppl. material, see www.karger.com/doi/10.1159/000498922). 


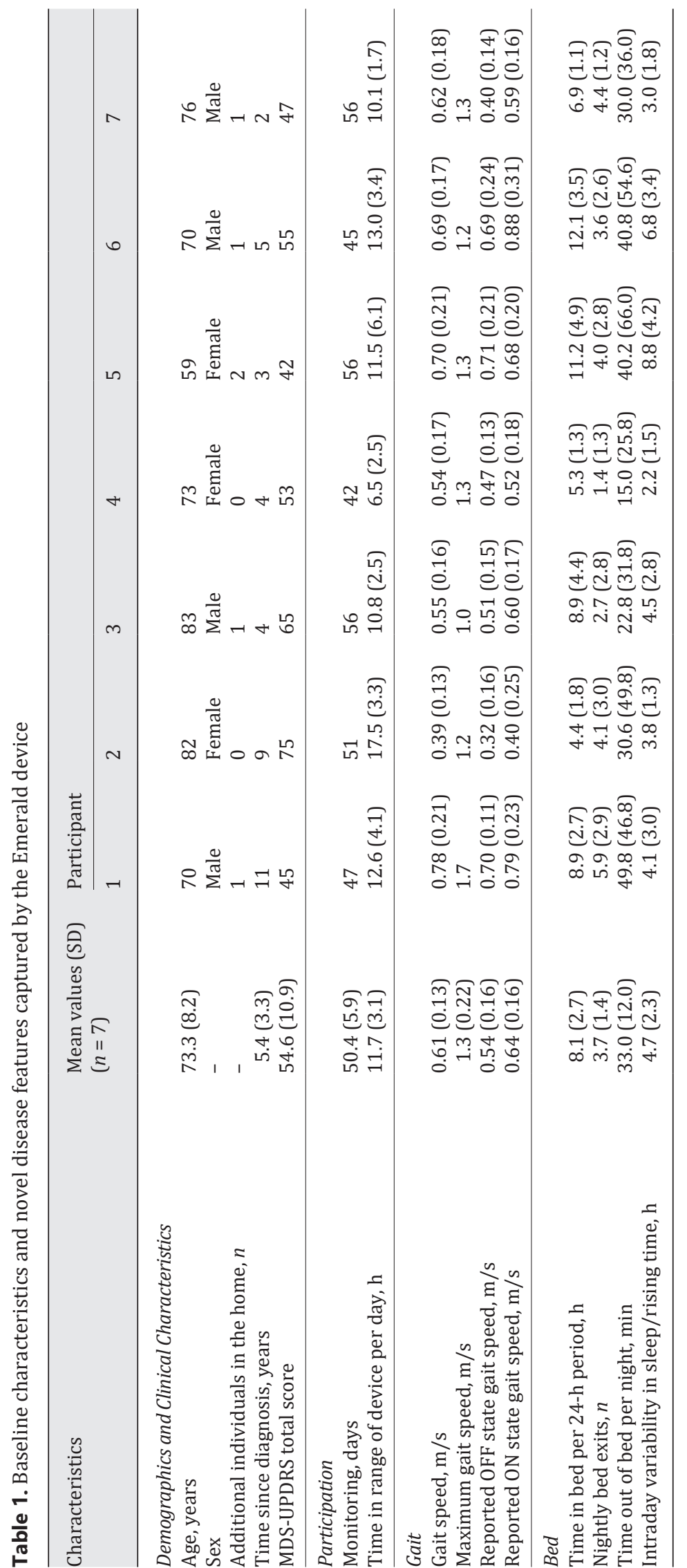


Kabelac et al.: Passive Monitoring at Home: A Pilot Study in Parkinson Disease

Finally, we assessed acceptance of monitoring with a survey, evaluating ease of participation, impact on daily activities, privacy concerns, and willingness to continue monitoring.

\section{Results}

We recruited 7 individuals with PD to have the Emerald device installed in their homes. All participants were white, and 6 (85.7\%) were college graduates. Mean (SD) disease duration among participants was 5.4 (3.3) years, and all participants were taking levodopa. Two individuals lived in an assisted-living facility (Participants 2 and 4); the remainder lived in freestanding homes. Additional baseline characteristics are presented in Table 1. We captured 353 days (90.1\%) of monitoring; the majority of missing data (29 days; $7.4 \%$ ) were due to lost WiFi connection.

Table 1 demonstrates the ability of the Emerald device to quantify mobility, time in bed, and a circadian schedule with variation seen across and within individuals. Gait speed varied by a factor of 2 (range $0.39-0.78 \mathrm{~m} / \mathrm{s}$ ) among the cohort. Mean time in bed per day (range 4.4-12.1 h), number (range 1.4-5.9) and duration (range 15.0-49.8 min) of nightly awakenings, and intraday variability of rest-activity rhythms (range $2.2-8.8 \mathrm{~h}$ ) also varied substantially.

Figure 2a demonstrates visualizations of activity over $3 \mathrm{~h}$ superimposed on a schematic of the home. Figure $2 \mathrm{~b}$ color-codes activity based on location in the space over the entire monitoring period. Figure 2c demonstrates a visualization of time in bed for 6 participants. Participants 4 and 7 had the lowest intraday variability in time in bed, generally spent the least time in bed, and had some of the least symptomatic (highest) scores on the PDSS. Conversely, Participants 5 and 6 had the most intraday variability in time in bed, spent the most time in bed, and had the lowest scores on the PDSS. Participant 5 , for example, spent an average of $11.2 \mathrm{~h}$ in bed per day and spent up to $20.5 \mathrm{~h}$ in bed in a single 24 -h period.

Device-derived gait speed had excellent correlation with the MDS-UPDRS total $(r=-0.88$, $p=0.009)$ and part III $(r=-0.95, p=0.001)$, with higher gait speed correlating with lower (less severely affected) scores. Mean device-derived gait speed increased significantly between Hauser-reported OFF and ON states $(p=0.025)$. The optimal averaging window to capture representative and reproducible gait speed measurements was 5 days. The derived minimal detectable change in mean gait speed over a 5-day period was $0.07 \mathrm{~m} / \mathrm{s}$ (online suppl. Material).

All participants agreed that participation was easy, and 6 agreed that their behavior was typical and that the device did not interfere with daily activity. Our participants had minimal privacy concerns, though 1 participant turned the device off for 10 days while a guest was visiting. Six participants indicated a willingness to participate in future monitoring. Figure 3 presents additional information regarding participant opinions on monitoring.

Fig. 2. Novel visualizations of participant activity at home and in bed derived from data collected by Emerald. a Movement visualizations superimposed on a schematic of the home (Participant 2). Left - continuous segment of motion over $3 \mathrm{~h}$. Right - heat map showing the proportion of time spent throughout the monitored space over 24 h. b Location in the space over the entire monitoring period for Participant 2. Each monitored day is represented as a concentric circle (day 1 at center, day 56 outside) with location around each circle representing time of day $(0=$ midnight $)$. $\mathbf{c}$ Time and motion in bed detected by Emerald among 6 participants. Each monitored day is represented as a concentric circle (day 1 at center, day 56 outside) with location around each circle representing time of day $(0=$ midnight $)$. Blue, time in bed; red, major change in position in bed (e.g., sitting up, arising); white, time out of bed. Mean time in bed per day and score on the Parkinson's Disease Sleep Scale (PDSS) are also shown for each participant.

(For figure see next page.) 

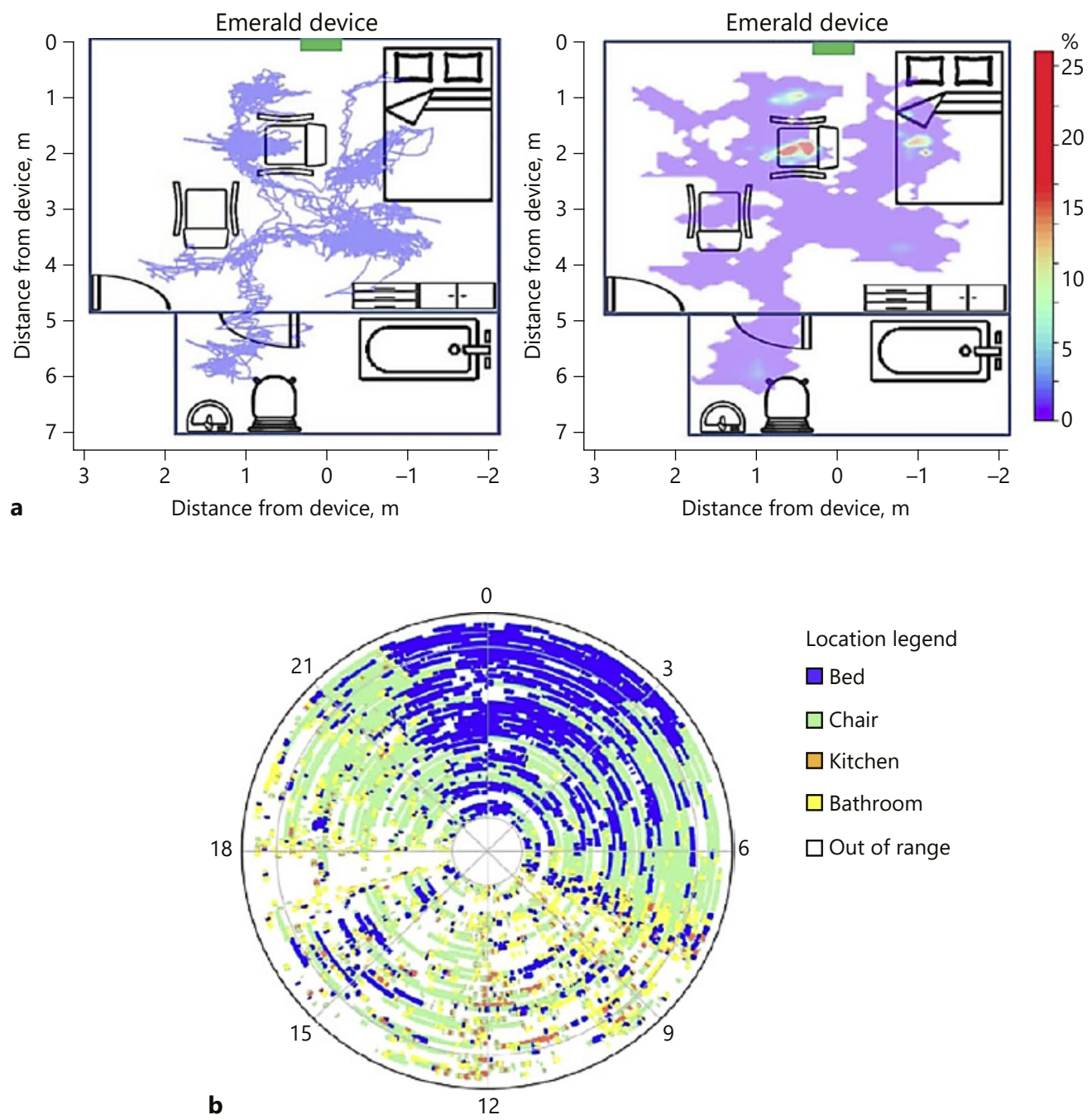

Location legend

$\square$ Bed

$\square$ Chair

$\square$ Kitchen

$\square$ Bathroom

$\square$ Out of range

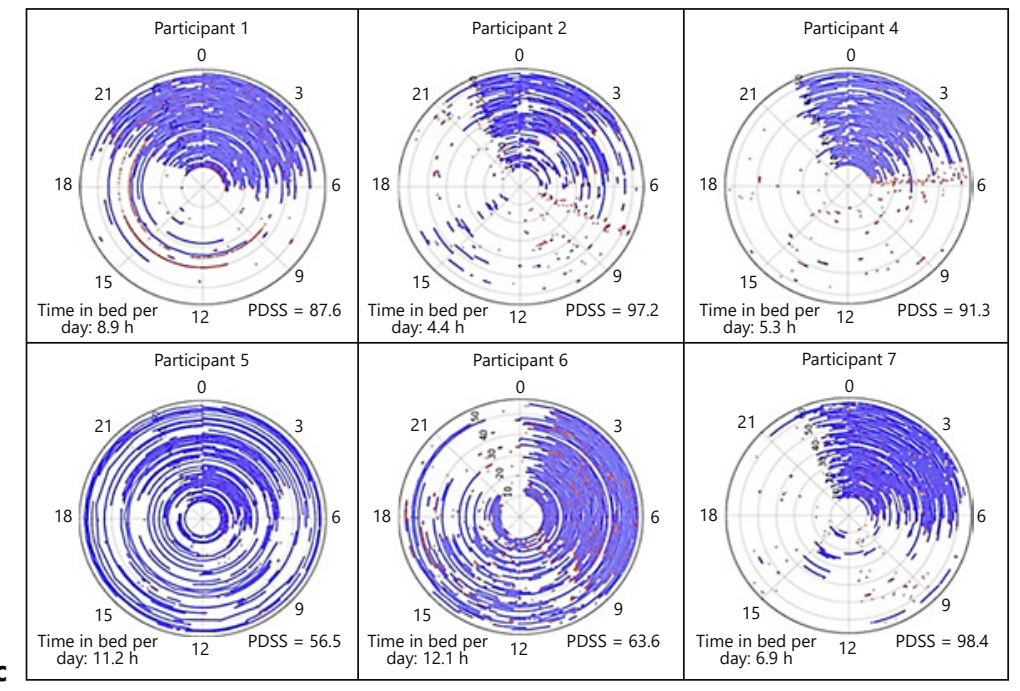




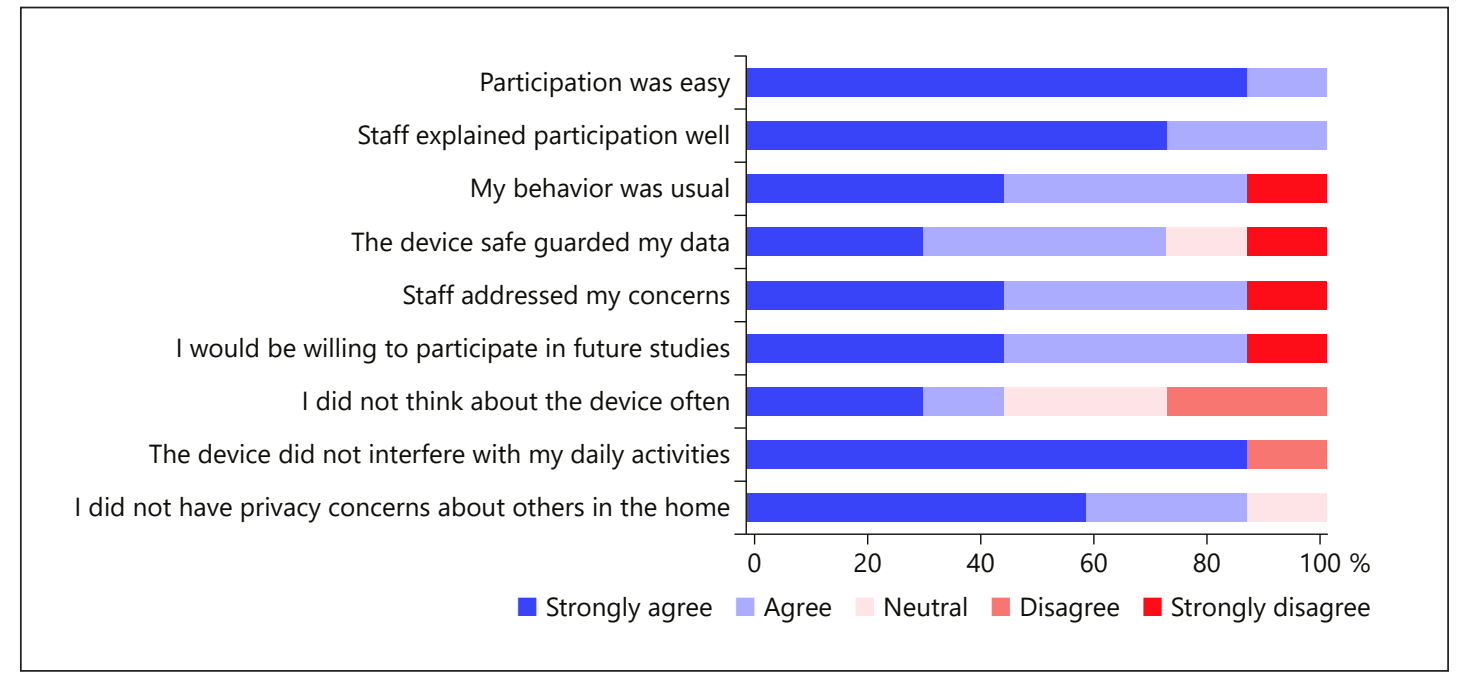

Fig. 3. Participant opinions on ease, safety, and privacy of monitoring using the Emerald device.

\section{Discussion}

Findings from this pilot study provide initial evidence of the ability to objectively characterize the home activities of individuals with PD using a passive monitoring system. Even in this small sample, we detected substantial variability in activity and time in bed within and across participants, observed that passively measured gait speed at home is slower than the measurement in the clinic [16], and assessed and visually represented patterns of daily activity and sleep. Device-derived measures correlate well with typical PD measures and offer a number of advantages. The device limits observational bias and complements office visits by providing insight into function at home. Additionally, unlike prior reports of home monitoring, our sensor can differentiate individuals in the home, allowing observation of a variety of environments $[1,2]$.

The Emerald device is sensitive to changes in gait speed over time. The 10-meter walk test, a clinic-based assessment of gait speed in PD, has a reported minimal detectable change of $0.22 \mathrm{~m} / \mathrm{s}$ [15], compared to $0.07 \mathrm{~m} / \mathrm{s}$ with the Emerald device. This increased sensitivity highlights the potential utility of the device in clinical research. The use of sensitive and objective outcome measures derived from novel technologies, like the Emerald device, could increase our ability to characterize the natural history of diseases and detect real changes in function in clinical trials.

This study has several limitations. The sample is small and not representative of the broader PD population. Additionally, portions of the home (including some bathrooms) were out-of-range of the device for most participants, and home environments differed significantly among participants. Regardless, we captured a large amount of data with 353 days and nights of observation from just 7 individuals. By contrast, some of the largest observational studies of sleep disorders in PD have included 100-200 nights of observation across the same number of participants $[17,18]$. Finally, the need for some participants to carry an accelerometer potentially introduced observational bias. We are currently developing a filter, unique to the consented individual, to eliminate the need for the accelerometer and address privacy concerns.

These pilot data provide unique insights into home function and suggest that activity is fragmented and variable in those with PD. Additionally, these data demonstrate that the 
Emerald device can generate outcome measures that may be more sensitive to change than traditional clinical outcome measures. Further data analysis and future studies in a larger cohort are necessary to validate measures derived in this pilot study, and to demonstrate the feasibility of evaluating other disease features (e.g., tremor, dyskinesias, or socialization) with the Emerald device. Additionally, we will compare the sensitivity of other Emeraldderived measures to traditional measures of PD severity.

Beyond PD, this technology may also be broadly applicable to other chronic conditions. For example, insights into patient sleep, home activities, socialization, and other observable behaviors could be invaluable in understanding the natural history of those with conditions from sickle cell disease to sleep apnea [3]. This technology has the potential to personalize healthcare, objectively monitor the effects of experimental therapeutics, and generate new insights into how chronic conditions affect people in the most important setting: their home.

\section{Statement of Ethics}

We obtained written consent from all participating individuals. The Institutional Review Boards at the University of Rochester and Massachusetts Institute of Technology approved all study procedures.

\section{Disclosure Statement}

Z.K., C.S., A.G., C.-Y.H., and R.H. report no disclosures related to this paper. C.G.T. has received honoraria for speaking at American Academy of Neurology courses; research support from the Michael J. Fox Foundation, American Academy of Neurology Institute, and the National Institute of Neurological Disorders and Stroke, National Institute of Health. B.F. has received research support from the Michael J. Fox Foundation. E.R.D. has received honoraria for speaking at American Academy of Neurology courses; received compensation for consulting activities from 23 and Me, Clintrex, GlaxoSmithKline, Grand Rounds, Lundbeck, MC10, MedAvante, Medico Legal services, the National Institute of Neurological Disorders and Stroke, Shire, Teva, and UCB; research support from AMC Health, Burroughs Wellcome Fund, Davis Phinney Foundation, Duke University, GlaxoSmithKline, Great Lakes Neurotechnologies, Greater Rochester Health Foundation, Huntington Study Group, the Michael J. Fox Foundation, National Science Foundation, Patient-Centered Outcomes Research Institute, Prana Biotechnology, Raptor Pharmaceuticals, Roche, Safra Foundation, and the University of California Irvine; and stock options from Grand Rounds. D.K. has received research support from the Michael J. Fox Foundation, Novartis, and LEO Pharma.

\section{Funding Sources}

This study was funded in part by the National Institute of Neurological Disorders and Stroke (P20 NS092529-02, P50 NS108676-01). 
Kabelac et al.: Passive Monitoring at Home: A Pilot Study in Parkinson Disease

\section{Author Contributions}

Z.K.: development of technology, study concept and design, acquisition of data, analysis and interpretation of data, drafting, and critical review of manuscript. C.G.T.: acquisition of data, analysis and interpretation of data, drafting, and critical review of manuscript. C.S.: acquisition of data, interpretation of data, and critical review of manuscript. B.F.: acquisition of data and interpretation of data. A.G.: acquisition of data, interpretation of data, and critical review of manuscript. C.-Y.H.: development of technology and analysis and interpretation of data. R.H.: development of technology and analysis and interpretation of data. E.R.D.: study concept and design, interpretation of data, critical review of manuscript, and study supervision. D.K.: development of technology, study concept and design, analysis and interpretation of data, critical review of manuscript, and study supervision.

\section{References}

1 Urwyler P, Stucki R, Rampa L, Müri R, Mosimann UP, Nef T. Cognitive impairment categorized in communitydwelling older adults with and without dementia using in-home sensors that recognise activities of daily living. Sci Rep. 2017 Feb;7(1):42084.

2 Dodge HH, Zhu J, Mattek NC, Austin D, Kornfeld J, Kaye JA. Use of High-Frequency In-Home Monitoring Data May Reduce Sample Sizes Needed in Clinical Trials. PLoS One. 2015 Sep;10(9):e0138095.

3 Dorsey ER, Papapetropoulos S, Xiong M, Kieburtz K. The First Frontier: digital biomarkers for neurodegenerative disorders. Digit Biomark. 2017;1(1):6-13.

4 Stone EE, Skubic M. Unobtrusive, continuous, in-home gait measurement using the Microsoft Kinect. IEEE Trans Biomed Eng. 2013 Oct;60(10):2925-32.

5 Austin D, Hayes TL, Kaye J, Mattek N, Pavel M. Unobtrusive monitoring of the longitudinal evolution of in-home gait velocity data with applications to elder care. In: 2011 Annual International Conference of the IEEE Engineering in Medicine and Biology Society. 2011;2011:6495-6498.

6 Adib F, Kabelac Z, Katabi D. Multi-Person Localization via RF Body Reflections. Paper presented at: NSDI2015. Available at: https://www.usenix.org/node/188986.

7 Hsu CY, Liu Y, Kabelac Z, Hristov R, Katabi D, Liu C. Extracting Gait Velocity and Stride Length from Surrounding Radio Signals. Proceedings of the 2017 CHI Conference on Human Factors in Computing Systems; 2017; Denver, Colorado, USA. https://doi.org/10.1145/3025453.3025937.

8 Adib F, Kabelac Z, Katabi D, Miller RC. 3D Tracking via Body Radio Reflections. Paper presented at: NSDI2014. Available at: https://www.usenix.org/node/179792.

9 Hauser RA, Friedlander J, Zesiewicz TA, Adler CH, Seeberger LC, O’Brien CF, et al. A home diary to assess functional status in patients with Parkinson's disease with motor fluctuations and dyskinesia. Clin Neuropharmacol. 2000 Mar-Apr;23(2):75-81.

10 Goetz CG, Tilley BC, Shaftman SR, Stebbins GT, Fahn S, Martinez-Martin P, et al.; Movement Disorder Society UPDRS Revision Task Force. Movement Disorder Society-sponsored revision of the Unified Parkinson's Disease Rating Scale (MDS-UPDRS): scale presentation and clinimetric testing results. Mov Disord. 2008 Nov; 23(15):2129-70.

11 Hoehn MM, Yahr MD. Parkinsonism: onset, progression and mortality. Neurology. 1967 May;17(5):427-42.

12 Chaudhuri KR, Pal S, DiMarco A, Whately-Smith C, Bridgman K, Mathew R, et al. The Parkinson's disease sleep scale: a new instrument for assessing sleep and nocturnal disability in Parkinson's disease. J Neurol Neurosurg Psychiatry. 2002 Dec; 73(6):629-35.

13 Hoops S, Nazem S, Siderowf AD, Duda JE, Xie SX, Stern MB, et al. Validity of the MoCA and MMSE in the detection of MCI and dementia in Parkinson disease. Neurology. 2009 Nov;73(21):1738-45.

14 Musiek ES, Bhimasani M, Zangrilli MA, Morris JC, Holtzman DM, Ju YS. Circadian Rest-Activity Pattern Changes in Aging and Preclinical Alzheimer Disease. JAMA Neurol. 2018 May;75(5):582-90.

15 Lang JT, Kassan TO, Devaney LL, Colon-Semenza C, Joseph MF. Test-Retest Reliability and Minimal Detectable Change for the 10-Meter Walk Test in Older Adults With Parkinson's disease. J Geriatr Phys Ther. 2016 Oct-Dec;39(4):165-70.

16 Hass CJ, Bishop M, Moscovich M, Stegemöller EL, Skinner J, Malaty IA, et al. Defining the clinically meaningful difference in gait speed in persons with Parkinson disease. J Neurol Phys Ther. 2014 0ct;38(4):233-8.

17 Postuma RB, Gagnon JF, Bertrand JA, Génier Marchand D, Montplaisir JY. Parkinson risk in idiopathic REM sleep behavior disorder: preparing for neuroprotective trials. Neurology. 2015 Mar;84(11):1104-13.

18 Jozwiak N, Postuma RB, Montplaisir J, Latreille V, Panisset M, Chouinard S, et al. REM Sleep Behavior Disorder and Cognitive Impairment in Parkinson's Disease. Sleep (Basel). 2017 Aug; 40(8). https://doi.org/10.1093/ sleep/zsx101. 